\title{
Pathways and roles of wall teichoic acid glycosylation in Staphylococcus aureus
}

DOI:

10.1016/j.ijmm.2013.10.009

\section{Document Version}

Accepted author manuscript

Link to publication record in Manchester Research Explorer

\section{Citation for published version (APA):}

Winstel, V., Xia, G., \& Peschel, A. (2014). Pathways and roles of wall teichoic acid glycosylation in Staphylococcus aureus. International Journal of Medical Microbiology, 304(3-4), 215-221.

https://doi.org/10.1016/j.ijmm.2013.10.009

\section{Published in:}

International Journal of Medical Microbiology

\section{Citing this paper}

Please note that where the full-text provided on Manchester Research Explorer is the Author Accepted Manuscript or Proof version this may differ from the final Published version. If citing, it is advised that you check and use the publisher's definitive version.

\section{General rights}

Copyright and moral rights for the publications made accessible in the Research Explorer are retained by the authors and/or other copyright owners and it is a condition of accessing publications that users recognise and abide by the legal requirements associated with these rights.

\section{Takedown policy}

If you believe that this document breaches copyright please refer to the University of Manchester's Takedown Procedures [http://man.ac.uk/04Y6Bo] or contact uml.scholarlycommunications@manchester.ac.uk providing relevant details, so we can investigate your claim.

\section{OPEN ACCESS}


Mini Review

\title{
Pathways and roles of wall teichoic acid glycosylation in Staphylococcus aureus
}

\author{
Volker Winstel $^{\mathrm{a}, \mathrm{b}}$, Guoqing Xia ${ }^{\mathrm{a}, \mathrm{b}, *}$, Andreas Peschel ${ }^{\mathrm{a}, \mathrm{b}}$ \\ a Cellular and Molecular Microbiology Division, Interfaculty Institute of Microbiology and Infection Medicine, University of Tübingen, \\ Elfriede-Aulhorn-Straße 6, 72076 Tübingen, Germany \\ ${ }^{\mathrm{b}}$ German Center for Infection Research (DZIF), partner site Tübingen, Germany
}

\section{A R T I C L E I N F O}

\section{Article history:}

Received 8 August 2013

Received in revised form 21 October 2013

Accepted 27 October 2013

Available online $\mathrm{xxx}$

\section{Keywords:}

S. aureus

Wall teichoic acid

Glycoepitopes

Bacteriophage

$\beta$-Lactam resistance

\begin{abstract}
A B S T R A C T
The thick peptidoglycan layers of Gram-positive bacteria are connected to polyanionic glycopolymers called wall teichoic acids (WTA). Pathogens such as Staphylococcus aureus, Listeria monocytogenes, or Enterococcus faecalis produce WTA with diverse, usually strain-specific structure. Extensive studies on $S$. aureus WTA mutants revealed important functions of WTA in cell division, growth, morphogenesis, resistance to antimicrobials, and interaction with host or phages. While most of the S. aureus WTA-biosynthetic genes have been identified it remained unclear for long how and why S. aureus glycosylates WTA with $\alpha-$ or $\beta$-linked N-acetylglucosamine (GlcNAc). Only recently the discovery of two WTA glycosyltransferases, TarM and TarS, yielded fundamental insights into the roles of $S$. aureus WTA glycosylation. Mutants lacking WTA GlcNAc are resistant towards most of the $S$. aureus phages and, surprisingly, TarS-mediated WTA $\beta$-O-GlcNAc modification is essential for $\beta$-lactam resistance in methicillin-resistant $S$. aureus. Notably, S. aureus WTA GlcNAc residues are major antigens and activate the complement system contributing to opsonophagocytosis. WTA glycosylation with a variety of sugars and corresponding glycosyltransferases were also identified in other Gram-positive bacteria, which paves the way for detailed investigations on the diverse roles of WTA modification with sugar residues.
\end{abstract}

(c) 2013 Elsevier GmbH. All rights reserved.

\section{Introduction}

The Gram-positive bacterium Staphylococcus aureus is one of the most successful opportunistic pathogens causing severe infections worldwide. The natural niche of $S$. aureus are the nares of healthy humans and $S$. aureus nasal carriage was shown to be a major risk factor for acquiring invasive infections (von Eiff et al., 2001).

Successful colonization of human nares and infection of tissues requires efficient mechanisms to counteract human defense systems and efficient attachment to human cells. One of the most important $S$. aureus nasal colonization factors is the cell wallbound wall teichoic acid (WTA) (Weidenmaier et al., 2004), a cell surface polymer produced by most Gram-positive bacteria including major human pathogens such as Streptococcus pneumoniae, Listeria monocytogenes or Enterococcus faecalis (Denapaite et al., 2012; Fiedler, 1988; Geiss-Liebisch et al., 2012). Interestingly, WTA structures are highly diverse among Gram-positive bacteria and are often strain or species-specific (Neuhaus and Baddiley,

\footnotetext{
* Corresponding author at: Cellular and Molecular Microbiology Division, Interfaculty Institute of Microbiology and Infection Medicine, University of Tübingen, Elfriede-Aulhorn-Straße 6, 72076 Tübingen, Germany. Tel.: +49 70712981514.

E-mail address: guoqing.xia@med.uni-tuebingen.de (G. Xia).
}

2003; Weidenmaier and Peschel, 2008). In most S. aureus clones including highly virulent strains such as USA300 and MW2 the WTA polymer consist of 11-40 ribitol-phosphate (RboP) repeating units substituted with D-alanine (D-ala) and $\mathrm{N}$-acetylglucosamine (GlcNAc). The WTA polymer is covalently linked to the $6-\mathrm{OH}$ group of $\mathrm{N}$-acetylmuramic acid residues in peptidoglycan via a disaccharide consisting of GlcNAc-1-P and N-actelymannosamine followed by two glycerol-phosphate (GroP) units (Weidenmaier and Peschel, 2008) (Fig. 1A). In addition to WTA S. aureus and most other Gram-positive bacteria produce membrane-bound lipoteichoic acids (LTA) whose backbone is composed of GroP repeating units (Reichmann and Grundling, 2011).

Major functional roles of $S$. aureus WTA were elucidated during the last couple of years using defined mutants with targeted deletion of WTA biosynthesis genes such as the ditABCD operon responsible for the D-alanylation of WTA (Peschel et al., 1999). Dala residues on WTA (and LTA) contribute to resistance to cationic antimicrobial peptides such as defensins or cathelicidins (Collins et al., 2002; Peschel et al., 1999) and to glycopeptide antibiotics such as vancomycin or teicoplanin (Peschel et al., 2000). Moreover, deletion of $\operatorname{tag} O$ encoding the first enzyme of the WTA biosynthesis pathway revealed that WTA is dispensable for $S$. aureus viability under laboratory conditions (Weidenmaier et al., 2004) but affects several critical cellular processes including autolysis 




B
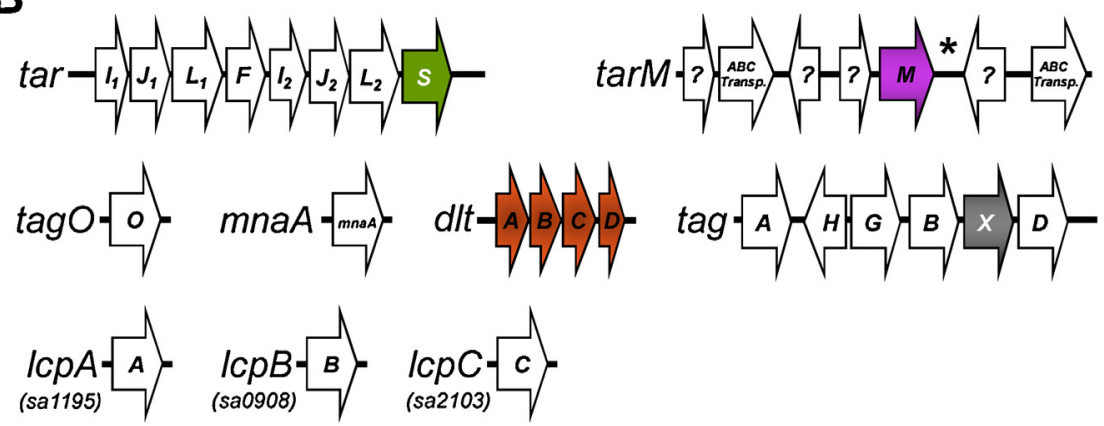

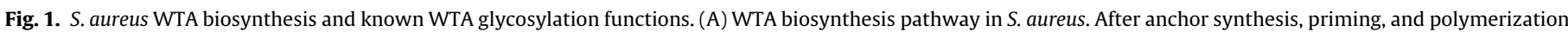

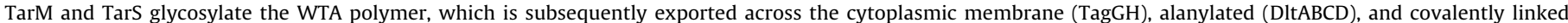

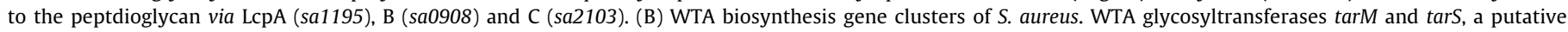

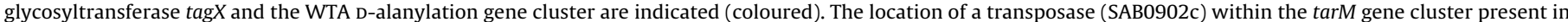

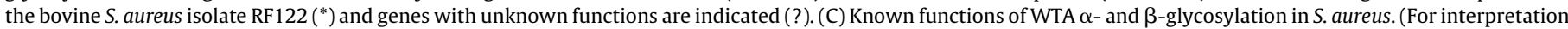
of the references to colour in this figure legend, the reader is referred to the web version of this article.)

(Vergara-Irigaray et al., 2008), cell division (Brown et al., 2012; Campbell et al., 2011), localization of penicillin binding protein 4 (PBP4) (Atilano et al., 2010), and survival at high temperatures (Vergara-Irigaray et al., 2008). Remarkably, S. aureus WTA contributes to biofilm formation (Vergara-Irigaray et al., 2008), adhesion to epithelial (Weidenmaier et al., 2004) and endothelial cells, and is important for severe invasive infections such as endocarditis (Weidenmaier et al., 2005). S. aureus WTA elicits robust antibody responses in humans and activates the human complement system via both the classical and the mannose-binding lectin pathway leading to opsonophagocytosis (Jung et al., 2012; Kurokawa et al., 2013; Park et al., 2010). How variation of WTA structure may affect the interaction of $S$. aureus with the innate and adaptive immune systems remains to be investigated.

During the last 60 years $S$. aureus increasingly acquired resistance genes to counteract extensive antibiotic treatment (Chambers and Deleo, 2009). Of note, WTAs are exposed at the $S$. aureus cell surface and they are required for PBP2a-mediated $\beta$ lactam antibiotic resistance (Campbell et al., 2011; Farha et al., 2013; Maki et al., 1994). Moreover, S. aureus mutants lacking WTA are susceptible to antimicrobial fatty acids from human skin (Kohler et al., 2009) and WTA contributes to S. aureus lysozyme resistance (Bera et al., 2007) suggesting a general protective role of WTA against antimicrobials. Because of its importance in vivo and in resistance to $\beta$-lactam antibiotics $S$. aureus WTA represents a very attractive target for the development of new antibiotics. Recently several promising novel compounds have been developed that inhibit TagO or the WTA transporter TagGH thereby rendering methicillin-resistant $S$. aureus (MRSA) susceptible to $\beta$-lactams (Campbell et al., 2011, 2012; Farha et al., 2013; Suzuki et al., 2011; Wang et al., 2013). Thus, WTA inhibitors could become useful for blocking $S$. aureus adhesion to host cells or for sensitizing MRSA to $\beta$-lactams.

Interaction with the human host during colonization or infection or the role of WTA in resistance towards antimicrobials are probably important reasons why $S$. aureus is synthesizing such a polymer. From an evolutionary point of view WTA seems to have a crucial function because WTA serve as adsorption receptor for many staphylococcal phages (Chatterjee, 1969; Park et al., 1974) that are largely responsible for horizontal gene transfer in Staphylococcus.

Although most of the $S$. aureus enzymes required for WTA biosynthesis have been elucidated and many functions have been attributed to WTA in the past years, it remained a mystery for decades how does $S$. aureus glycosylate WTA. Of note, early studies revealed strain-specific anomeric configurations ( $\alpha$ - and $\beta$ ) of the WTA GlcNAc substitutions (Endl et al., 1984) suggesting that $S$. aureus may encode two different WTA glycosyltransferases.

\section{In silico analysis of WTA glycosyltransferases in S. aureus genomes}

Most of the genes and enzymes involved in the initiation, (tagOA, mnaA), priming $(\operatorname{tag} D B)$, polymerization ( $\operatorname{tar} I J L F)$, transport 
(tagGH), and D-alanylation (dltABCD) of S. aureus WTA were identified and studied in genetic and/or in vitro reconstitution approaches (Swoboda et al., 2010; Xia et al., 2010a). Recently the ligation of WTA from lipid-linked WTA precursors to peptidoglycan was proposed to be accomplished by a new family of enzymes named LCP proteins (Chan et al., 2013; Dengler et al., 2012; Kawai et al., 2011) (Fig. 1A and B). However, it remained unknown until recently, which genes encode the WTA glycosyltransferases in S. aureus (Fig. 1B).

S. aureus NCTC8325-4 encodes ca. 20 putative glycosyltransferases and interestingly, two putative glycosyltransferases are co-localized within well studied WTA biosynthesis gene clusters namely tarS and $\operatorname{tag} X$ (Brown et al., 2012; Swoboda et al., 2010; Xia et al., 2010a) (Fig. 1B). In contrast, the WTA glycosyltransferase tarM (Xia et al., 2010b) does not co-localize with WTA gene clusters but is adjacent to genes encoding putative $A B C$ transporters and proteins of unknown function (Fig. 1B). Remarkably TarM does not exists in every $S$. aureus genome and seem to be strain specific (see below).

\section{The two unrelated enzymes TarM and TarS glycosylate WTA of $S$. aureus}

Evidence for WTA glycosylation by $\beta$-GlcNAc in $S$. aureus dates back to the 1960 s and 1970s. WTA $\beta$-GlcNAc transferase activity was demonstrated in strain Copenhagen (Nathenson and Strominger, 1963) while a phage-resistant mutant 52B2 was found to lack any detectable WTA GlcNAc transferase activity (Shaw et al., 1970). By screening a transposon mutant library derived from S. aureus strain RN4220, a mutant that was resistant to serogroup B phages was isolated and named K6. Sequencing analysis revealed that the transposon had disrupted a gene (SACOL1042), which encodes a putative glycosyltransferase. The gene product was named TarM. Based on the facts that mutant K6 that bears a transposon insertion in tarM lacked GlcNAc on WTAs and tarM complemented K6 mutant synthesizes $\alpha$-O-GlcNAc on WTA, it was concluded that TarM is a WTA $\alpha-O$-GlcNAc transferase (Xia et al., 2010b). Surprisingly, unlike transposon mutant K6, a targeted tarM deletion mutant (S. aureus RN4220 $\Delta$ tarM) was still phage-susceptible and nuclear magnetic resonance (NMR) elucidation revealed the presence of $\beta-O-G l c N A c$ substituted WTA in this strain further suggesting $S$. aureus encodes a second WTA GlcNAc transferase, potentially tagX or tarS (Brown et al., 2012). However, combined deletion of tarM and tagX did not alter phage susceptibility (Xia et al., unpublished data) and no in vitro WTA glycosyltransferase activity was found for TagX (Brown et al., 2012). When the other putative $\beta$-glycosyltransferase gene tarS of mutant K6 was sequenced it was found to be truncated because of a point mutation. Moreover, TarS glycosylated RboP WTA polymers in vitro and deletion of both tarM and tarS resulted in complete absence of GlcNAc strongly suggesting tarS encodes the second S. aureus WTA glycosyltransferase (Brown et al., 2012).

Thus, TarM and TarS represent the RboP WTA $\alpha$ - and $\beta-O-$ GlcNAc transferases, respectively, of S. aureus (Fig. 1A and B).

\section{Functional roles of WTA glycosylation in S. aureus}

While the discovery of TarM and TarS clarified the molecular basis of WTA glycosylation several questions remained unanswered: (i) Why is $S$. aureus glycosylating its WTA, (ii) are there different functions for $\alpha$ - or $\beta$-O-GlcNAc residues linked to WTA, and (iii) how is WTA glycosylation regulated? Several early studies already suggested that WTA GlcNAc may serve as an adsorption receptor for serogroup B phages such as $\Phi 52$ A (Chatterjee, 1969; Park et al., 1974). However, S. aureus mutants lacking either $\alpha$ - or
$\beta$-O-GlcNAc remained susceptible to most of the $S$. aureus phages while only the complete loss of WTA glycosylation resulted in phage resistance (Brown et al., 2012; Xia et al., 2010b, 2011; Xia and Wolz, 2013) (Table 1 and Fig. 1C). Thus, serogroup B phages require WTA glycosylation for efficient adsorption and infection but do not distinguish between different anomeric configurations of the GlcNAc residues on WTA. Similar observations were made for horizontal transfer of $S$. aureus pathogenicity islands (SaPIs) by SaPI particles formed by helper phage proteins indicating that SaPI particles adopt the receptor specificities of helper phages and do not distinguish between different WTA GlcNAc configurations (Winstel et al., 2013). Remarkably, similar WTA structures enable horizontal gene transfer among Gram-positive pathogenic bacteria, even across long phylogenetic distances (Winstel et al., 2013) (Table 1 and Fig. 1C).

MRSA mutants lacking WTA were found to be highly susceptible to $\beta$-lactam antibiotics, such as methicillin (Campbell et al., 2011; Farha et al., 2013). In contrast to MRSA mutants lacking either the D-alanine or $\alpha-O$-GlcNAc substitutions on WTA, which were still resistant to most $\beta$-lactam antibiotics the lack of $\beta$-O-GlcNAc residues rendered MRSA susceptible to $\beta$-lactams (Brown et al., 2012) (Table 1 and Fig. 1C). The reasons for the essential role of TarS in $\beta$-lactam resistance remain elusive. The WTA $\beta$-O-GlcNAc may serve as a scaffold for the cell wall synthesis complex or cell wall-associated proteins that directly or indirectly modulate PBP2a activity (Brown et al., 2012). Along this line there is evidence that PBP2a and PBP4 interact with WTA (Atilano et al., 2010; Qamar and Golemi-Kotra, 2012). However, it is also possible that WTA glycosylation affects the physicochemical properties of the peptidoglycan matrix by influencing the cell wall milieu (Sutcliffe, 2012).

Mutants lacking $\beta$-O-GlcNAc were also more susceptible to lysostaphin, which cleaves the pentaglycine crossbridges of $S$. aureus peptidoglycan, than the parental strains (Brown et al., 2012) suggesting that WTA $\beta$-O-GlcNAc interferes with binding of lysostaphin to peptidoglycan (Fig. 1C). In contrast, several other critical processes that depend on WTA such as autolysis, biofilm formation, and cell division were not affected in mutants lacking WTA glycosylation (Brown et al., 2012) (Fig. 1C). Interestingly, there are first indications that $S$. aureus can modulate the relative amounts of $\alpha$ - and $\beta$-glycosylation depending on environmental conditions. tarS but not tarM expression levels were strongly upregulated by oxacillin treatment, which underscores the critical role of TarS in $\beta$-lactam resistance (Brown et al., 2012).

Human sera contain high levels of antibodies directed against the $\beta$-GlcNAc residues of $S$. aureus WTA indicating that these modifications can be dominant antigens in staphylococcal infections (Kurokawa et al., 2013). Moreover, human MBL was found to bind to glycosylated WTA and activated the lectin pathway of complement (Kurokawa et al., 2013) (Table 1 and Fig. 1C).

It remains unclear, which $S$. aureus regulation system controls the WTA glycosylation pattern and if there are further conditions by which tarM or tarS expression is affected. Thus, WTA glycosylation plays crucial roles in the susceptibility of $S$. aureus to phages, $\beta$-lactam antibiotics, lysostaphin and interaction with the human immune system.

\section{tarM is absent in several $S$. aureus clonal complexes}

While tarS was found in nearly all so far sequenced $S$. aureus genomes tarM is absent in several strains (Table 2). In particular tarM seems to be an ancient feature of $S$. aureus because the very early branching $S$. aureus clonal complex (CC) 75 , sequence type (ST) 1850 strain MSHR1132 bears both, tarS and tarM in its genome (Holt et al., 2011) (Table 2). Interestingly, tarM is absent in several healthcare-associated MRSA strains such as N315, Mu50, Mu3, JH1 
Table 1

WTA structures and known WTA glycosylation functions in various Gram-positive bacteria.

\begin{tabular}{|c|c|c|}
\hline Species/strain & WTA type ${ }^{\mathrm{a}, \mathrm{b}}$ (glycosylation) & $\begin{array}{l}\text { Phenotype of WTA glycosylation-deficient mutants } \\
\text { (reference) }\end{array}$ \\
\hline S. aureus RN4220 & $\operatorname{RboP}(\alpha, \beta-G l c N A c)$ & $\begin{array}{l}\text { Phage resistance; increased lysostaphin and } \beta \text {-lactam } \\
\text { susceptibility in MRSA (Brown et al., 2012; Xia et al., } \\
\text { 2010b, 2011); decreased SaPI particle susceptibility } \\
\text { (Winstel et al., 2013); no MBL mediated complement } \\
\text { activation, no anti-WTA IgG binding, no C3 and C4 } \\
\text { deposition (Kurokawa et al., 2013) }\end{array}$ \\
\hline S. epidermidis ATCC14990 & $\operatorname{GroP}(\alpha-G l c, \alpha-G l c N A c)$ & No mutants described \\
\hline B. subtilis 168 & $\operatorname{GroP}(\alpha-G l c)$ & Phage resistance (Allison et al., 2011; Young, 1967) \\
\hline B. subtilis W23 & $\operatorname{RboP}(\beta-G l c)$ & unknown \\
\hline L. monocytogenes Serotype $1 / 2 \mathrm{a}$ & RboP ( $\alpha$-GlcNAc, $\alpha$-Rha) & $\begin{array}{l}\text { No binding of CDBP35 }{ }^{\mathrm{d}} \text { (Eugster et al., 2011), phage } \\
\text { resistance (Tran et al., 1999) }\end{array}$ \\
\hline L. monocytogenes serotype $4 \mathrm{~b}$ & $\operatorname{RboP}($ Glc, Gal) & $\begin{array}{l}\text { Phage resistance (Cheng et al., 2008); attenuated virulence } \\
\text { in gastrointestinal listeriosis (mice) (Faith et al., 2009) }\end{array}$ \\
\hline L. grayi ATCC27840 & RboP (GlcNAc) & No mutants described \\
\hline E. faecalis V583 & $\begin{array}{l}\text { GalNAc-RboP }(\alpha-\text { Rha }) \text { (WTA } 1) \text {; } \\
\text { Glc-GalNAc-RboP }(\alpha-\text { Glc })(\text { WTA } 2)\end{array}$ & No mutants described \\
\hline E. faecium U0317 & GalNAc-GroP (absent) & No WTA glycosylation \\
\hline S. pneumonia & $\begin{array}{l}\text { AATGal-Glc-RboP-GalNAc-GalNAc } \\
\text { (GalNAc }{ }^{\mathrm{e}} \text { \& P-Cho) }\end{array}$ & $\begin{array}{l}\text { No mutants described; phosphocholine moieties } \\
\text { contribute to virulence (Gehre et al., 2009; Kharat and } \\
\text { Tomasz, 2006) }\end{array}$ \\
\hline
\end{tabular}

a WTA repeating units (bold) and WTA glycosylation ( ) are indicated.

b Ribitol-phosphate (RboP), glycerol-phosphate (GroP), N-acetylglucosamine (GlcNAc), N-acetylgalactosamine (GalNAc), glucose (Glc), Rhamnose (Rha), Galactose (Gal), 2-acetamido-4-amino-2,4,6-trideoxygalactose (AATGal) and phosphorylcholine (P-Cho) are indicated.

c Strain V583 synthesizes two different WTAs.

d CDBP35, cell wall binding domain of Listeria phage endolysin PlyP35.

e A recent study by Gisch et al. (2013) suggest no GalNAc modification on LTA and potentially on WTA because of identical repeating units of LTA and WTA.

(all CC5) or certain ST398 strains (Table 2). As indicated in Table 2 there was no correlation between the presence or absence of tarM and the MRSA status but the absence of tarM seems to be a conserved feature of certain STs or even of several closely related STs.
In addition, there was no correlation of tarM with host specificity, e.g. human or bovine origin (e.g. USA300 or RF122, respectively; both bear tarM) (Table 2). It can be assumed that the loss of tarM happened repeatedly but only rarely during $S$. aureus evolution.

Table 2

Presence of the tarM and tarS genes in available S. aureus genomes.

\begin{tabular}{|c|c|c|c|c|c|}
\hline S. aureus strain ${ }^{\mathrm{a}}$ & $\operatorname{tar} S^{\mathrm{b}}$ & $\operatorname{tar} M^{\mathrm{b}, \mathrm{c}}$ & MRSA status & Sequence type & Clonal complex \\
\hline MW2* & + & + & MRSA & 1 & 1 \\
\hline MSSA476* & + & + & MSSA & 1 & 1 \\
\hline TCH70 & + & + & MRSA & 1 & 1 \\
\hline N315* & + & - & MRSA & 5 & 5 \\
\hline Mu50* & + & - & MRSA & 5 & 5 \\
\hline Mu3* & + & - & MRSA & 5 & 5 \\
\hline A5937 & + & - & MRSA & 5 & 5 \\
\hline A6224 & + & - & MRSA & 5 & 5 \\
\hline $\mathrm{JH} 1^{*}$ & + & - & MRSA & 105 & 5 \\
\hline 04-02981 & + & $\sim$ & MRSA & 225 & 5 \\
\hline USA300* & + & + & MRSA & 8 & 8 \\
\hline NCTC8325* & + & + & MSSA & 8 & 8 \\
\hline RN4220 & + & + & MSSA & 8 & 8 \\
\hline Newman* & + & + & MSSA & 8 & 8 \\
\hline TW20* & + & + & MRSA & 239 & $8(239)$ \\
\hline $\mathrm{COL}^{*}$ & + & + & MRSA & 250 & 8 \\
\hline Н0-5096-0412* & + & $\sim$ & MRSA & 22 & 22 \\
\hline TCH60 & + & + & MRSA & 30 & 30 \\
\hline MN8 & + & + & MSSA & 30 & 30 \\
\hline C101 & + & + & MSSA & 30 & 30 \\
\hline BTN1260 & + & + & MSSA & 30 & 30 \\
\hline MRSA252* & + & + & MRSA & 36 & 30 \\
\hline A9635 & + & - & MSSA & 45 & 45 \\
\hline MSHR1132 & + & + & MSSA & 1850 & 75 \\
\hline RF122* & + & + & MSSA & 151 & 151 \\
\hline PS187 & - & - & MSSA & 395 & 395 \\
\hline S0385* & + & $\sim$ & MRSA & 398 & 398 \\
\hline 71193 & + & $\sim$ & MSSA & 398 & 398 \\
\hline DR10 & + & - & MSSA & 398 & 398 \\
\hline
\end{tabular}

a Only fully annotated genomes are indicated $\left({ }^{*}\right)$.

b Presence $(+)$ or absence $(-)$ of tarS or tarM genes are indicated.

c Truncated tarM variant is indicated $(\sim)$. 
Currently it is mysterious where tarM came from during $S$. aureus evolution and if tarM is mobile. tarM may have been acquired via horizontal gene transfer since tarM is flanked by a transposase possibly contributing to mobility e.g. in the bovine $S$. aureus isolate RF122 (Table 2 and Fig. 1B). In addition, it remains unclear if there is any advantage of bearing or losing tarM and if $\alpha$-glycosylated WTA is beneficial for certain S. aureus lineages during colonization or infection or for susceptibility to certain phages.

\section{Role of WTA glycosylation in other Gram-positive bacteria}

Most of the Gram-positive bacteria have WTAs of highly variable composition and structure at their cell surfaces. Even among staphylococcal species WTA structures are quite diverse (Endl et al., 1984). While $S$. aureus WTA is composed of RboP units most of the coagulase-negative staphylococci (CoNS) produce GroP WTAs or WTA polymers with complex composition containing e.g. hexoses in the WTA backbones (Endl et al., 1984). Most WTAs of CoNS are glycosylated with sugars other than GlcNAc that is found in most $S$. aureus (Endl et al., 1984) (Table 1). Thus, bearing tarM and tarS and especially $\beta-O-G$-GNAc WTA glycosylation seems to be a specific feature of $S$. aureus raising the question why CoNS use different glycosylation patterns and which genes are involved. Of note early studies revealed that certain $S$. aureus produce WTA structures other than the common RboP polymer, e.g. S. aureus strains 187 and 406, which were found to produce GroP WTA substituted with $\alpha$-O-linked N-acetylgalactosamine (GalNAc) (Endl et al., 1984). Recently such a GroP type WTA structure was verified in S. aureus strain PS187 by using NMR spectroscopy (Winstel et al., 2013). It seems that those $S$. aureus strains with GroP WTA may be more related to CoNS in terms of cell envelope structure than to classical S. aureus strains.

WTAs produced by other Gram-positive bacteria are in some instances similar to $S$. aureus WTA but most were shown to be highly diverse (Neuhaus and Baddiley, 2003; Weidenmaier and Peschel, 2008). Extensive investigations elucidated WTA structure, function, and biosynthesis in Bacillus subtilis. B. subtilis strain 168 produces GroP WTA substituted with $\alpha$-linked glucose (Glc) while strain W23 produced RboP WTA modified with $\beta$-Glc (Brown et al., 2012; Neuhaus and Baddiley, 2003; Swoboda et al., 2010) (Table 1). Decades ago it was shown that glucosylated WTA were required for phage adsorption in B. subtilis 168 (Young, 1967) (Table 1) and the TagE protein (also called GtaA and RodD) of B. subtilis 168 was proposed as the WTA glucosyltransferase based on the indirect genetic evidence (Honeyman and Stewart, 1989; Mauel et al., 1989). Recently, TagE was characterized and demonstrated to be a GroP WTA $\alpha$-glucosyl transferase (Allison et al., 2011). Thus, WTA glycosylation in B. subtilis and S. aureus share similar functions such as phage adsorption and infection. In addition TarQ was recently found to act as a $\beta$-O-glucosyl transferase in $B$. subtilis strain W23 producing RboP WTA substituted with $\beta$-Glc (Brown et al., 2012).

Human pathogens from the genus Listeria including certain serotypes of $L$. monocytogenes or L. grayi produce WTA with RboP repeating units identical to those found in S. aureus (Fiedler, 1988). Often their WTA glycosylation pattern is also shared with $S$. aureus but additional sugars such as rhamnose or Glc were described (Fiedler, 1988) (Table 1). Studies on WTA glycosylation in Listeria revealed functions related to those of $S$. aureus. WTA glycosyl residues of $L$. monocytogenes serve as adsorption receptors for different Listeria phages (Cheng et al., 2008; Tran et al., 1999) and can contribute to virulence, e.g. to gastrointestinal listeriosis in mice (Faith et al., 2009) (Table 1). In agreement with these findings the cell wall binding domain (CBD) of Listeria phage endolysin PlyP35 was found to recognize the GlcNAc residues on WTA (Eugster et al., 2011) (Table 1). However, this property does not seem to be a common feature of all Listeria phage endolysins (Eugster and
Loessner, 2012). WTA was also shown to restrict the access of certain phage endolysin CBDs to the peptidoglycan of $L$. monocytogenes (Eugster and Loessner, 2012). In contrast Enterococcus spp. WTAs have a very complex structure, which is distinct from that of $S$. aureus or Listeria. Of note, E. faecalis or E. faecium produce different WTAs with hexose-containing repeating units (GalNAc-RboP glycosylated with $\alpha$-linked rhamnose or Glc-GalNAc-RboP glycosylated with $\alpha$-linked Glc, both WTAs found in E. faecalis V583 whereas non-substituted GalNAc-GroP, type WTA was found in $E$. faecium U0317) (Bychowska et al., 2011; Geiss-Liebisch et al., 2012) (Table 1). Although some functions of Enterococcus WTAs including evasion from the lectin pathways of the human complement system have recently been described (Geiss-Liebisch et al., 2012) it still has to be elucidated how and why enterococci glycosylate their WTA. Similar to enterococci streptococci such as Streptococcus pneumoniae synthesize very complex polymers with identical repeating units in WTA and LTA, which are glycosylated with GalNAc (Denapaite et al., 2012) (Table 1) although recent studies on S. pneumoniae LTA suggest no any glycosylation, possibly its WTA was not glycosylated either (Gisch et al., 2013). Additionally S. pneumoniae teichoic acids bear unusual phosphocholine residues that play crucial roles in S. pneumoniae virulence (Gehre et al., 2009; Kharat and Tomasz, 2006). Recent studies on Lactobacillus plantarum WTA revealed strain-specific WTA types, including both GroP and RboP WTA polymers substituted with diverse $\alpha$-D-glucosyl residues (Tomita et al., 2012). Interestingly, L. plantarum seems to be capable of WTA-switching between WTA types with different repeating units ('alditol switching') (Bron et al., 2012; Tomita et al., 2013). Thus, although most Gram-positive bacteria produce WTA, structure and glycosylation patterns of the polymers are highly diverse. Further investigation on the diversity of WTA and characterization of mutants with altered WTA will disclose more functions of WTA glycosylation.

\section{Perspectives}

While the $S$. aureus WTA biosynthesis pathway including the WTA glycosylation process is largely known now (Brown et al., 2012; Xia et al., 2010b) it remains mysterious why S. aureus glycosylates its WTA with GlcNAc in two different anomeric configurations. So far only a few functional roles of $S$. aureus WTA glycosylation were identified and the elucidation of staphylococcal WTA glycosylation paves the way for dissecting the protein-carbohydrate interactions in Staphylococcus-host interaction. Especially the function of $\alpha-O-G$-GNAc-modified WTA and the significance of strain-dependent presence or absence of tarM remains unclear. Thus, the elucidation of mechanisms regulating $S$. aureus WTA glycosylation especially under in vivo conditions will help to understand why two different enzymatic pathways exist and how they may contribute to fitness or virulence. Moreover, exploring if and which WTA glycosyl residues are expressed in vivo will be important for vaccination approaches with WTA-specific opsonic antibodies.

During the last few years $S$. aureus WTA biosynthetic enzymes became promising targets for novel antibiotics because MRSA strains become highly susceptible to $\beta$-lactam antibiotics when lacking WTA or WTA $\beta-O-G l c N A c$ (Brown et al., 2012; Campbell et al., 2011; Farha et al., 2013). Crystal structures of WTA biosynthesis proteins as reported recently for $S$. epidermidis TagF (Lovering et al., 2010), S. aureus TagD (Fong et al., 2006), or B. cereus DltA (Osman et al., 2009) will help in the rational design of new antimicrobial drug.

It is still mysterious why the various Gram-positive bacteria are using completely different WTA biosynthesis pathways and why so many different WTA structures exist. Amazingly a common feature of various phages infecting distantly related Gram-positive bacteria 
is their requirement for WTA as adsorption receptor. Since phages are probably crucial vehicles for horizontal gene transfer of virulence and resistance genes the elucidation of the underlying 'WTA 'glycocode' will enable to predict the likeliness of future phagemediated horizontal gene transfer events, and to better understand the evolution of Gram-positive bacteria.

\section{Acknowledgments}

We thank our coworkers and collaborators for helpful discussion and support. We apologize to those whose work is not cited in this article. Our work is supported by grants from the German Research Council (DFG) TRR34 to A.P. and SFB766 to A.P. and G.X. and grants from the German Center for Infection Research (DZIF) to A.P. and G.X.

\section{References}

Allison, S.E., D'Elia, M.A., Arar, S., Monteiro, M.A., Brown, E.D., 2011. Studies of the genetics, function, and kinetic mechanism of TagE, the wall teichoic acid glycosyltransferase in Bacillus subtilis 168. The Journal of Biological Chemistry 286, 23708-23716

Atilano, M.L., Pereira, P.M., Yates, J., Reed, P., Veiga, H., Pinho, M.G., Filipe, S.R., 2010. Teichoic acids are temporal and spatial regulators of peptidoglycan cross-linking in Staphylococcus aureus. Proceedings of the National Academy of Sciences of the United States of America 107, 18991-18996.

Bera, A., Biswas, R., Herbert, S., Kulauzovic, E., Weidenmaier, C., Peschel, A., Gotz, F., 2007. Influence of wall teichoic acid on lysozyme resistance in Staphylococcus aureus. Journal of Bacteriology 189, 280-283.

Bron, P.A., Tomita, S., van, S., Remus, I.I., Meijerink, D.M., Wels, M., Okada, M., Wells, S., Kleerebezem, J.M., 2012. Lactobacillus plantarum possesses the capability for wall teichoic acid backbone alditol switching. Microbial Cell Factories 11, 123.

Brown, S., Xia, G., Luhachack, L.G., Campbell, J., Meredith, T.C., Chen, C., Winstel, V., Gekeler, C., Irazoqui, J.E., Peschel, A., et al., 2012. Methicillin resistance in Staphylococcus aureus requires glycosylated wall teichoic acids. Proceedings of the National Academy of Sciences of the United States of America.

Bychowska, A., Theilacker, C., Czerwicka, M., Marszewska, K., Huebner, J., Holst, O., Stepnowski, P., Kaczynski, Z., 2011. Chemical structure of wall teichoic acid isolated from Enterococcus faecium strain U0317. Carbohydrate Research 346, 2816-2819.

Campbell, J., Singh, A.K., Santa Maria Jr., J.P., Kim, Y., Brown, S., Swoboda, J.G., Mylonakis, E., Wilkinson, B.J., Walker, S., 2011. Synthetic lethal compound combinations reveal a fundamental connection between wall teichoic acid and peptidoglycan biosyntheses in Staphylococcus aureus. ACS Chemical Biology 6, 106-116.

Campbell, J., Singh, A.K., Swoboda, J.G., Gilmore, M.S., Wilkinson, B.J., Walker, S., 2012. An antibiotic that inhibits a late step in wall teichoic acid biosynthesis induces the cell wall stress stimulon in Staphylococcus aureus. Antimicrobial Agents and Chemotherapy 56, 1810-1820.

Chambers, H.F., Deleo, F.R., 2009. Waves of resistance: Staphylococcus aureus in the antibiotic era. Nature Reviews Microbiology 7, 629-641.

Chan, Y.G., Frankel, M.B., Dengler, V., Schneewind, O., Missiakas, D., 2013. Staphylococcus aureus mutants lacking the LytR-CpsA-Psr family of enzymes release cell wall teichoic acids into the extracellular medium. Journal of Bacteriology 195, 4650-4659.

Chatterjee, A.N., 1969. Use of bacteriophage-resistant mutants to study the nature of the bacteriophage receptor site of Staphylococcus aureus. Journal of Bacteriology 98, 519-527

Cheng, Y., Promadej, N., Kim, J.W., Kathariou, S., 2008. Teichoic acid glycosylation mediated by gtcA is required for phage adsorption and susceptibility of Listeria monocytogenes serotype 4b. Applied and Environmental Microbiology 74, 1653-1655

Collins, L.V., Kristian, S.A., Weidenmaier, C., Faigle, M., Van Kessel, K.P., Van Strijp, J.A., Gotz, F., Neumeister, B., Peschel, A., 2002. Staphylococcus aureus strains lacking D-alanine modifications of teichoic acids are highly susceptible to human neutrophil killing and are virulence attenuated in mice. The Journal of Infectious Diseases 186, 214-219.

Denapaite, D., Bruckner, R., Hakenbeck, R., Vollmer, W., 2012. Biosynthesis of teichoic acids in Streptococcus pneumoniae and closely related species: lessons from genomes. Microbial Drug Resistance 18, 344-358.

Dengler, V., Meier, P.S., Heusser, R., Kupferschmied, P., Fazekas, J., Friebe, S., Staufer, S.B., Majcherczyk, P.A., Moreillon, P., Berger-Bachi, B., et al., 2012. Deletion of hypothetical wall teichoic acid ligases in Staphylococcus aureus activates the cell wall stress response. FEMS Microbiology Letters 333, 109-120.

Endl, J., Seidl, P.H., Fiedler, F., Schleifer, K.H., 1984. Determination of cell wall teichoic acid structure of staphylococci by rapid chemical and serological screening methods. Archives of Microbiology 137, 272-280.

Eugster, M.R., Haug, M.C., Huwiler, S.G., Loessner, M.J., 2011. The cell wall binding domain of Listeria bacteriophage endolysin PlyP35 recognizes terminal GlcNAc residues in cell wall teichoic acid. Molecular Microbiology 81, 1419-1432.
Eugster, M.R., Loessner, M.J., 2012. Wall teichoic acids restrict access of bacteriophage endolysin Ply118, Ply511, and PlyP40 cell wall binding domains to the Listeria monocytogenes peptidoglycan. Journal of Bacteriology 194, 6498-6506.

Faith, N., Kathariou, S., Cheng, Y., Promadej, N., Neudeck, B.L., Zhang, Q., Luchansky, J., Czuprynski, C., 2009. The role of L. monocytogenes serotype 4b gtcA in gastrointestinal listeriosis in A/J mice. Foodborne Pathogens and Disease 6, 39-48.

Farha, M.A., Leung, A., Sewell, E.W., D'Elia, M.A., Allison, S.E., Ejim, L., Pereira, P.M., Pinho, M.G., Wright, G.D., Brown, E.D., 2013. Inhibition of WTA synthesis blocks the cooperative action of PBPs and sensitizes MRSA to beta-lactams. ACS Chemical Biology 8, 226-233.

Fiedler, F., 1988. Biochemistry of the cell surface of Listeria strains: a locating general view. Infection 16 (Suppl. 2), S92-S97.

Fong, D.H., Yim, V.C., D’Elia, M.A., Brown, E.D., Berghuis, A.M., 2006. Crystal structure of CTP:glycerol-3-phosphate cytidylyltransferase from Staphylococcus aureus: examination of structural basis for kinetic mechanism. Biochimica et Biophysica Acta 1764, 63-69.

Gehre, F., Spisek, R., Kharat, A.S., Matthews, P., Kukreja, A., Anthony, R.M., Dhodapkar, M.V., Vollmer, W., Tomasz, A., 2009. Role of teichoic acid choline moieties in the virulence of Streptococcus pneumoniae. Infection and Immunity 77, 2824-2831.

Geiss-Liebisch, S., Rooijakkers, S.H., Beczala, A., Sanchez-Carballo, P., Kruszynska, K. Repp, C., Sakinc, T., Vinogradov, E., Holst, O., Huebner, J., et al., 2012. Secondary cell wall polymers of Enterococcus faecalis are critical for resistance to complement activation via mannose-binding lectin. The Journal of Biological Chemistry 287, 37769-37777.

Gisch, N., Kohler, T., Ulmer, A.J., Muthing, J., Pribyl, T., Fischer, K., Lindner, B., Hammerschmidt, S., Zahringer, U., 2013. Structural reevaluation of Streptococcus pneumoniae Lipoteichoic acid and new insights into its immunostimulatory potency. The Journal of Biological Chemistry 288, 15654-15667.

Holt, D.C., Holden, M.T., Tong, S.Y., Castillo-Ramirez, S., Clarke, L., Quail, M.A., Currie, B.J., Parkhill, J., Bentley, S.D., Feil, E.J., et al., 2011. A very early-branching Staphylococcus aureus lineage lacking the carotenoid pigment staphyloxanthin. Genome Biology and Evolution 3, 881-895.

Honeyman, A.L., Stewart, G.C., 1989. The nucleotide sequence of the rodC operon of Bacillus subtilis. Molecular Microbiology 3, 1257-1268.

Jung, D.J., An, J.H., Kurokawa, K., Jung, Y.C., Kim, M.J., Aoyagi, Y., Matsushita, M., Takahashi, S., Lee, H.S., Takahashi, K., et al., 2012. Specific serum Ig recognizing staphylococcal wall teichoic acid induces complement-mediated opsonophagocytosis against Staphylococcus aureus. Journal of Immunology 189, 4951-4959.

Kawai, Y., Marles-Wright, J., Cleverley, R.M., Emmins, R., Ishikawa, S., Kuwano, M. Heinz, N., Bui, N.K., Hoyland, C.N., Ogasawara, N., et al., 2011. A widespread family of bacterial cell wall assembly proteins. The EMBO Journal 30, 4931-4941.

Kharat, A.S., Tomasz, A., 2006. Drastic reduction in the virulence of Streptococcus pneumoniae expressing type 2 capsular polysaccharide but lacking choline residues in the cell wall. Molecular microbiology 60, 93-107.

Kohler, T., Weidenmaier, C., Peschel, A., 2009. Wall teichoic acid protects Staphylococcus aureus against antimicrobial fatty acids from human skin. Journal of Bacteriology 191, 4482-4484.

Kurokawa, K., Jung, D.J., An, J.H., Fuchs, K., Jeon, Y.J., Kim, N.H., Li, X., Tateishi, K. Park, J.A., Xia, G., et al., 2013. Glycoepitopes of staphylococcal wall teichoic acid govern complement-mediated opsonophagocytosis via human serum antibody and mannose-binding lectin. The Journal of Biological Chemistry 288 (43), 30956-30968.

Lovering, A.L., Lin, L.Y., Sewell, E.W., Spreter, T., Brown, E.D., Strynadka, N.C., 2010 Structure of the bacterial teichoic acid polymerase TagF provides insights into membrane association and catalysis. Nature Structural \& Molecular Biology 17, 582-589.

Maki, H., Yamaguchi, T., Murakami, K., 1994. Cloning and characterization of a gene affecting the methicillin resistance level and the autolysis rate in Staphylococcus aureus. Journal of Bacteriology 176, 4993-5000.

Mauel, C., Young, M., Margot, P., Karamata, D., 1989. The essential nature of teichoic acids in Bacillus subtilis as revealed by insertional mutagenesis. Molecular \& General Genetics: MGG 215, 388-394.

Nathenson, S.G., Strominger, J.L., 1963. Enzymatic synthesis of nacetylglucosaminylribitol linkages in teichoic acid from staphylococcus aureus, strain copenhagen. The Journal of Biological Chemistry 238, 3161-3169.

Neuhaus, F.C., Baddiley, J., 2003. A continuum of anionic charge: structures and functions of D-alanyl-teichoic acids in gram-positive bacteria. Microbiology and Molecular Biology Reviews: MMBR 67, 686-723.

Osman, K.T., Du, L., He, Y., Luo, Y., 2009. Crystal structure of Bacillus cereus D-alany carrier protein ligase (DltA) in complex with ATP. Journal of Molecular Biology $388,345-355$.

Park, J.T., Shaw, D.R., Chatterjee, A.N., Mirelman, D., Wu, T., 1974. Mutants of staphylococci with altered cell walls. Annals of the New York Academy of Sciences 236, 54-62.

Park, K.H., Kurokawa, K., Zheng, L., Jung, D.J., Tateishi, K., Jin, J.O., Ha, N.C., Kang H.J., Matsushita, M., Kwak, J.Y., et al., 2010. Human serum mannose-binding lectin senses wall teichoic acid Glycopolymer of Staphylococcus aureus, which is restricted in infancy. The Journal of Biological Chemistry 285, 27167-27175.

Peschel, A., Otto, M., Jack, R.W., Kalbacher, H., Jung, G., Gotz, F., 1999. Inactivation of the dlt operon in Staphylococcus aureus confers sensitivity to defensins, protegrins, and other antimicrobial peptides. The Journal of Biological Chemistry 274 8405-8410.

Peschel, A., Vuong, C., Otto, M., Gotz, F., 2000. The D-alanine residues of Staphylococcus aureus teichoic acids alter the susceptibility to vancomycin and the activity of autolytic enzymes. Antimicrobial Agents and Chemotherapy 44, 2845-2847. 
Qamar, A., Golemi-Kotra, D., 2012. Dual roles of FmtA in Staphylococcus aureus cell wall biosynthesis and autolysis. Antimicrobial Agents and Chemotherapy 56, 3797-3805.

Reichmann, N.T., Grundling, A., 2011. Location, synthesis and function of glycolipids and polyglycerolphosphate lipoteichoic acid in Gram-positive bacteria of the phylum Firmicutes. FEMS Microbiology Letters 319, 97-105.

Shaw, D.R., Mirelman, D., Chatterjee, A.N., Park, J.T., 1970. Ribitol teichoic acid synthesis in bacteriophage-resistant mutants of Staphylococcus aureus $\mathrm{H}$. The Journal of Biological Chemistry 245, 5101-5106.

Sutcliffe, I.C., 2012. Exposing a chink in the armor of methicillin-resistant Staphylococcus aureus. Proceedings of the National Academy of Sciences of the United States of America 109, 18637-18638.

Suzuki, T., Swoboda, J.G., Campbell, J., Walker, S., Gilmore, M.S., 2011. In vitro antimicrobial activity of wall teichoic acid biosynthesis inhibitors against Staphylococcus aureus isolates. Antimicrobial Agents and Chemotherapy 55, 767-774.

Swoboda, J.G., Campbell, J., Meredith, T.C., Walker, S., 2010. Wall teichoic acid function, biosynthesis, and inhibition. Chembiochem: A European Journal of Chemical Biology 11, 35-45.

Tomita, S., de Waard, P., Bakx, E.J., Schols, H.A., Kleerebezem, M., Bron, P.A., 2013. The structure of an alternative wall teichoic acid produced by a Lactobacillus plantarum WCFS1 mutant contains a 1,5-linked poly(ribitol phosphate) backbone with 2-alpha-D-glucosyl substitutions. Carbohydrate Research 370, 67-71.

Tomita, S., Furihata, K., Tanaka, N., Satoh, E., Nukada, T., Okada, S., 2012. Determination of strain-specific wall teichoic acid structures in Lactobacillus plantarum reveals diverse alpha-D-glucosyl substitutions and high structural uniformity of the repeating units. Microbiology 158, 2712-2723.

Tran, H.L., Fiedler, F., Hodgson, D.A., Kathariou, S., 1999. Transposon-induced mutations in two loci of Listeria monocytogenes serotype 1/2a result in phage resistance and lack of $\mathrm{N}$-acetylglucosamine in the teichoic acid of the cell wall. Applied and Environmental Microbiology 65, 4793-4798.

Vergara-Irigaray, M., Maira-Litran, T., Merino, N., Pier, G.B., Penades, J.R., Lasa, I., 2008. Wall teichoic acids are dispensable for anchoring the PNAG exopolysaccharide to the Staphylococcus aureus cell surface. Microbiology 154, 865-877.

von Eiff, C., Becker, K., Machka, K., Stammer, H., Peters, G., 2001. Nasal carriage as a source of Staphylococcus aureus bacteremia, Study Group. The New England Journal of Medicine 344, 11-16.
Wang, H., Gill, C.J., Lee, S.H., Mann, P., Zuck, P., Meredith, T.C., Murgolo, N., She X., Kales, S., Liang, L., et al., 2013. Discovery of wall teichoic acid inhibitors as potential anti-MRSA beta-lactam combination agents. Chemistry \& Biology 20, 272-284.

Weidenmaier, C., Kokai-Kun, J.F., Kristian, S.A., Chanturiya, T., Kalbacher, H., Gross, M., Nicholson, G., Neumeister, B., Mond, J.J., Peschel, A., 2004. Role of teichoic acids in Staphylococcus aureus nasal colonization, a major risk factor in nosocomial infections. Nature Medicine 10, 243-245.

Weidenmaier, C., Peschel, A., 2008. Teichoic acids and related cell-wall glycopolymers in Gram-positive physiology and host interactions. Nature Reviews Microbiology 6, 276-287.

Weidenmaier, C., Peschel, A., Xiong, Y.Q., Kristian, S.A., Dietz, K., Yeaman, M.R., Bayer, A.S., 2005. Lack of wall teichoic acids in Staphylococcus aureus leads to reduced interactions with endothelial cells and to attenuated virulence in a rabbit model of endocarditis. The Journal of Infectious Diseases 191, 17711777.

Winstel, V., Liang, C., Sanchez-Carballo, P., Steglich, M., Munar, M., Broker, B.M., Penades, J.R., Nubel, U., Holst, O., Dandekar, T., et al., 2013. Wall teichoic acid structure governs horizontal gene transfer between major bacterial pathogens. Nature Communications 4, 2345.

Xia, G., Corrigan, R.M., Winstel, V., Goerke, C., Grundling, A., Peschel, A., 2011. Wall teichoic acid-dependent adsorption of staphylococcal siphovirus and myovirus. Journal of Bacteriology 193, 4006-4009.

Xia, G., Kohler, T., Peschel, A., 2010a. The wall teichoic acid and lipoteichoic acid polymers of Staphylococcus aureus. International Journal of Medical Microbiology: IJMM 300, 148-154

Xia, G., Maier, L., Sanchez-Carballo, P., Li, M., Otto, M., Holst, O., Peschel, A., 2010b. Glycosylation of wall teichoic acid in Staphylococcus aureus by TarM. The Journal of Biological Chemistry 285, 13405-13415.

Xia, G., Wolz, C., 2013. Phages of Staphylococcus aureus and their impact on host evolution. Infection, Genetics and Evolution: Journal of Molecular Epidemiology and Evolutionary Genetics in Infectious Diseases.

Young, F.E., 1967. Requirement of glucosylated teichoic acid for adsorption of phage in Bacillus subtilis 168. Proceedings of the National Academy of Sciences of the United States of America 58, 2377-2384. 